Anwachsen des Resonanzintegrals $\beta_{12}$ in nematischer Phase von $0,90 \beta_{0}$ auf $0,95 \beta_{0}$ ermöglicht werden.

Das beobachtete Maximum im Temperaturverlauf von $\Delta a\left(\mathrm{C}_{9}\right)$ läßt sich qualitativ ebenfalls durch eine Spindichte-Umverteilung verstehen. Wegen der Kleinheit des Effektes und wegen der Komplikation, die durch die Abhängigkeit der isotropen $\mathrm{C}_{9}{ }^{13}$-Hfs-Konstante auch von benachbarten Spindichten hervorgeru- fen wird, schien uns aber eine quantitative Rechnung nicht lohnend.

Der Deutschen Forschungsgemeinschaft danken wir für die Bereitstellung von Meßgeräten und für die Gewährung eines Forschungsstipendiums für H. H. Herrn R. BIEHL danken wir für seine experimentelle Hilfe, Herrn W. Kaminski für einige Spindichte-Berechnungen.

\section{Nuclear Magnetic Relaxation in a Dilute Gas of Spherical Rotors}

\section{IRVING OZIER}

Institute of Astronomy and Space Science University of British Columbia, Vancouver 8, Canada

(Z. Naturforsch. 26 a, 1232-1233 [1971]; received 23 January 1971)

In a recent Article ${ }^{1}$ in this Journal, McCourt and Hess presented a theoretical treatment of nuclear spin relaxation in a dilute gas of spherical rotors for the case where the relaxation mechanism is governed by the spin-rotation interaction $W_{\mathrm{SR}}$. Unlike the earlier analyses ${ }^{2,3}$, the McCourt-Hess calculation focuses on effects associated with the symmetry properties of the molecules and attempts to take these into account. Unfortunately, in deriving a convenient form for $W_{\mathrm{SR}}$, serious errors were made in treating these molecular symmetry properties with the result that the arguments upon which the symmetry-related conclusions rest are not valid. It is the purpose of this Note to point out these errors and to discuss briefly some of the associated implications. We select $\mathrm{CH}_{4}$ as a representative spherical rotor and confine most of our detailed remarks to this example.

MH do not attempt to present a complete analysis. They consider only effects associated with "zero-frequency" transitions. These are transitions which conserve both the energy of rigid rotation and the energy of centrifugal distortion ${ }^{4}$, so that their frequencies are, in magnitude, $\lesssim\left|\omega_{0}\right|$, where $\omega_{0}$ is the nuclear larmor frequency. The $\mathrm{MH}$ analysis is based on the assumption that only such zerofrequency transitions need be considered, and it is within this framework that the current criticism is presented.

MH specifically exclude effects associated with two types of "high-frequency" transitions. In the first, the rotational quantum number is not conserved and the energy of rigid rotation is changed. In the second, the total nuclear spin quantum number $I$ is not conserved

1 F. R. McCourt and S. Hess, Z. Naturforsch. 25 a, 1169 [1970].

2 M. Bloom, F. Bridges, and W. N. Hardy, Can. J. Phys. 45, 3533 [1967]

3 J. S. Blicharski, Physica 39, 161 [1968].

4 P. N. Yi, I. Ozier, and C. H. Anderson, Phys. Rev. 165, 92 [1968]. This work is referred to as Paper I.

5 The fact that a change in I implies a change in the centrifugal distortion energy is discussed in Sect. III.A of $\mathrm{Pa}$ - and the energy of centrifugal distortion is changed ${ }^{5}$. The justification for neglecting these transitions ${ }^{6}$ lies in the fact that they occur at frequencies whose magnitude is, in general, $\gg\left|\omega_{0}\right|$.

It should be pointed out that there is a third type of highfrequency transition in which $I$ is conserved but the centrifugal distortion energy is not. The possibility of such transitions has not been mentioned previously in the literature. However, because of their high frequency, they are also excluded from the framework of the MH treatment and the present discussion.

Of the many symmetry-related conclusions derived by $\mathrm{MH}$, only one representative result will be quoted here. In Eq. (4.10) of MH, it is shown that, for the limit of extreme narrowing, the spin-lattice relaxation time $T$ is given by

$$
\frac{1}{T}=\frac{2}{3}\left\langle J^{2}\right\rangle_{0}\left\{\frac{c_{\mathrm{a}}^{2}}{\omega_{\text {coll }}}+\frac{q c_{\mathrm{d}^{2}}}{45 \tilde{\omega}_{\text {coll }}}\right\} .
$$

Here $c_{\mathrm{a}}$ is the average spin rotation constant and $c_{\mathrm{d}}$ is the anisotropy in the spin rotation matrix. $\left\langle J^{2}\right\rangle_{0}$ is the average value of $J(J+1)$. The collision integrals $\omega_{\text {coll }}$ and $\tilde{\omega}_{\text {coll }}$ are defined, respectively, in Eqs. (3.11) and (3.12) of MH. For tetrahedral molecules, $q=3$; for octahedral molecules, $q=2$ (for values of $J>2$ ). It is one of the major conclusions of $\mathrm{MH}$ that $T$ depends in this manner on the symmetry of the particular spherical rotor under consideration.

Because we are confining our detailed remarks to $\mathrm{CH}_{4}$, we can make direct use of the work of YI, OzIER and ANDERson (Paper I) ${ }^{4}$ and of OzIer, Crapo and LEE (Paper II) ${ }^{7}$. These two papers present (among other things) a detailed group theoretical treatment of (1) the spin rotation interaction $W_{\mathrm{SR}}$; (2) the necessary spin-rotational wave functions and (3) all the matrix elements required for an analysis which considers only zero-frequency transitions ${ }^{8}$.

In Sec. III.A of Paper I, there is defined a representation $\Gamma$ which takes into account all the symmetry requirements which the wave functions must satisfy. Al-

per I. Notice that this fact is true only for the tetrahedral molecule $\mathrm{XY}_{4}$ if the spin of nucleus $\mathrm{Y}$ is $1 / 2$. It is not true for other tetrahedral molecules or any octahedral molecules.

${ }^{6}$ It is understood that one is also neglecting transitions in which both $I$ and $J$ change.

7 I. Ozier, L. M. Crapo, and S. S. Lee, Phys. Rev. 172. 63 [1968]. This work is referred to as Paper II.

8 Note that $e_{i} e_{i}(i=1,2,3,4)$ in $\mathrm{MH}$ is $-\frac{1}{3} \varepsilon^{i}$ in Refs. ${ }^{4,}$ ?. 
though MH do not explicitly introduce any representation, their arguments implicitly assume one, and it is clear that this assumed representation is not equivalent to $\Gamma$. This selection of an inappropriate representation is the fundamental error in the $\mathrm{MH}$ treatment and leads to three errors in the detailed development of the effective spin rotation operator given in Eq. (2.13) of $\mathrm{MH}$. We shall consider each of these in turn.

(1) The total nuclear spin $I$ is a good quantum number $^{9}$ in a dilute gas and can equal 2,1 or 0 . The constant $c_{\mathrm{d}}$ contributes only to the relaxation of the $(I=1)$ species and not to the relaxation of the $(I=2)$ species ${ }^{4}$. No account of this fact is taken in $\mathrm{MH}$.

(2) While the MH treatment clearly fails for the $(I=2)$ species, it may still be valid for the $(I=1)$ case. However, for this case, it can be shown that the simplification of Eq. (2.9) of $\mathrm{MH}$ is not correct. This equation can be written

$$
I^{\prime}=L_{1}+L_{2}
$$

where $\quad L_{1}=-\frac{1}{2}\left[I^{(1)}+I^{(2)}+I^{(3)}\right] \cdot \overline{e_{4} e_{4}}$

and $\quad L_{2}=-\frac{1}{2}\left[I^{(1)} \cdot \overline{e_{2} e_{2}}+I^{(2)} \cdot \overline{e_{1} e_{1}}+I^{(3)} \cdot \overline{e_{3} e_{3}}\right]$.

If we use the notation of Papers I and II, then

$$
\begin{aligned}
& L_{1}=L_{\mathrm{A}}+L_{\mathrm{B}} \\
& \text { and } \quad L_{2}=L_{\mathrm{A}}-L_{\mathrm{B}} \text {, } \\
& \text { where } \quad L_{\mathrm{A}}=-\frac{1}{6} \sum_{\xi=x y z} I^{\xi} \cdot \varepsilon^{\xi} \\
& \text { and } \quad L_{\mathrm{B}}=-\frac{1}{6}\left[I^{x} \cdot\left(\varepsilon^{y}+\varepsilon^{z}\right)+I^{y} \cdot\left(\varepsilon^{z}+\varepsilon^{x}\right)+I^{z}\right. \\
& \left.\cdot\left(\varepsilon^{x}+\varepsilon^{y}\right)\right] \text {. }
\end{aligned}
$$

It can be shown ${ }^{7}$ by simple group theory (or by direct evaluation) that, in representation $\Gamma, L_{\mathrm{B}} \cdot J$ has no nonzero matrix elements diagonal in $I$. Since transitions offdiagonal in $I$ are being neglected, we can, within the context of the present problem, write

$$
L_{1}=L_{2} \text {. }
$$

Yet in MH, $L_{2}$ is set equal to zero and only $L_{1}$ is retained.

(3) The MH calculation can be modified so as to take Eq. (7) into account simply by multiplying $c_{\mathrm{d}}$ by a factor of two. However, the analysis leading from Eq. (2.12) to Eq. (2.13) of $\mathrm{MH}$ is also incorrect. The operator

$$
H_{\mathrm{SR}} \equiv \widetilde{e_{4} e_{4}} \cdot \mathrm{J}
$$

plays the role of a spin-rotational magnetic field. $\mathrm{MH}$

9 It should be kept in mind that we are referring here specifically to $\mathrm{CH}_{4}$.

10 While MH originally referred $K$ to the "4" axis, MH subsequently showed that $K$ (as defined in $\mathrm{MH}$ ) could equally well be referred to any arbitrary axis fixed in the molecule. Thus, for example, $K$ can be associated with the $Z$ axis of the molecule-fixed frame shown in Fig. 1 of Paper I. This particular choice is the most convenient one for comparing MH to Papers I and II. argue that $\tilde{l} \cdot H_{\mathrm{SR}}$ has non-zero matrix elements diagonal in $J$ only for the component of $H_{\mathrm{SR}}$ along $J$. They then apply arguments equivalent to the use of a simple vector model to obtain

where

$$
H_{\mathrm{SR}}=\alpha J,
$$

$$
\alpha=\left(\frac{K^{2}}{J(J+1)}-\frac{1}{3}\right) .
$$

Here $K$ is the quantum number associated with the projection of $J$ on the " 4 " axis defined MH.

However, when the matrix elements of $\tilde{I} \cdot a J$ are evaluated in representation $\Gamma$, it is found that they all vanish. This results is not surprising since $\tilde{I} \cdot a J$ has only non-zero matrix elements diagonal in $K$, whereas the complete expression for the tensor part of the spin rotation interaction has only non-zero matrix elements off-diagonal in $K$, as can be seen from Eq. (A4) of Paper II ${ }^{10}$. An assumption equivalent to Eq. (9) was made by GORDON ${ }^{11}$ in analysing the magnetic-resonance molecular-beam spectrum of $\mathrm{CH}_{4}$ observed by ANDERSON and RAMSEY ${ }^{12}$ and was, in part, responsible for the fact that the Gordon analysis gave incorrect results ${ }^{7}$.

It is clear from arguments (1), (2) and (3) presented here that the expressions [e. g. Eq. (1) above] for the spin-lattice relaxation times derived in $\mathrm{MH}$ for tetrahedral molecules are not correct. Although we have not discussed the octahedral case here, it is felt that a reexamination of this part of the problem is in order as well.

In Sec. 5 of $\mathrm{MH}$, the data available in the literature on $c_{\mathrm{d}}$ for $\mathrm{CH}_{4}$ is reviewed in the light of the $\mathrm{MH}$ treatment and it is concluded that the best value for $\left|c_{\mathrm{d}}\right|$ is $21.0 \mathrm{kHz}{ }^{12,7}$, rather than $18.2 \mathrm{kHz}^{13}$. It is clear from our remarks here that the basis for this conclusion is not valid. In fact, the best current value for $c_{d}$ is $+(18.5 \pm 0.5) \mathrm{kHz}$, as obtained by YI, OzIER, and RAMSEY 14.

In spite of the difficulties in the MH treatment, this work represents a significant step forward. Besides pointing out the possibility that symmetry considerations may be important, they extend the kinetic equation approach to the problem of NMR in polyatomic molecules and show many of the advantages of this technique over the correlation function approach. When the treatment of $W_{\mathrm{SR}}$ has been modified so as to include the symmetry requirements correctly, the $\mathrm{MH}$ calculation will be very helpful in the analysis of spinlattice relaxation data on spherical rotors.

The authors wishes to thank Professor M. Bцoom for his many helpful comments.

11 R. G. Gordon, J. Chem. Phys. 44, 1184 [1966].

12 C. H. Anderson and N. F. Ramsey, Phys. Rev. 149, 14 [1966].

13 P. N. Yi, I. Ozier, A. Khosla, and N. F. Ramsey, Bull. Amer. Phys. Soc. 12, 509 [1967].

14 P. N. YI, I. Ozier, and N. F. Ramsey, Hyperfine Spectrum of $\mathrm{CH}_{4}$ (to be published in J. Chem. Phys.). 